\title{
Exame Clínico da Osteoartrose do Joelho com Realidade Aumentada
}

\author{
Nigel da Silva Lima ${ }^{1}$, Anselmo Cardoso de Paiva ${ }^{1}$, Raul Frankllim ${ }^{1}$, \\ Geraldo Braz Júnior ${ }^{1}$, Daniel Lima ${ }^{1}$, Humberto Oliveira Serra ${ }^{1}$, \\ Lucas Bezerra Maia ${ }^{1}$ \\ ${ }^{1}$ Universidade Federal do Maranhão (UFMA) \\ Av. dos Portugueses, 1966 - Vila Bacanga, São Luís - MA, 65080-805 \\ \{nigel, paiva, geraldo, daniel, lucas.maia\}@nca.ufma.br,
dr.raulalmeida@gmail.com, humberto.serra@huufma.br
}

\begin{abstract}
Knee Osteoarthrosis (OA) is a degenerative disease that contributes greatly to reduced mobility and loss of functional independence. Usually, the short-size radiograph is used to estimate the angle of deviation that forms on the atanomic axis of the knee. In this work, we present the development of an augmented reality tool that assists in the measurement of this deviation, in a clinical way. We evaluated 27 knees from 15 patients, which the measurements were compared with the measurements obtained by the proposed method. The value obtained in the comparison of the measures shows a strongly significant agreement, with ICC $=0.972$, validating the methodology as a measurement alternative.
\end{abstract}

Resumo. A osteoartrose (OA) do joelho é uma doença degenerativa que contribui grandemente para a redução de mobilidade e perda de independência funcional. Comumente, utiliza-se a radiografia de tamanho curto para estimação do ângulo de desvio que se forma no eixo atanômico do joelho. Neste trabalho, é apresentado o desenvolvimento de uma ferramenta de realidade aumentada (RA) que auxilia na mensuração desse desvio, de forma clínica. Foram avaliados 27 joelhos de 15 pacientes, onde as mensurações por meio de radiografia foram comparadas com as medidas obtidas pelo método proposto. $O$ valor obtido na comparação das medidas apresenta concordância fortemente significativa, com ICC $=0.972$, validando a metodologia como alternativa de mensuração.

\section{Introdução}

A osteoartrose (OA) do joelho, doença de caráter degenerativo, é uma das formas mais comuns de artrose, sendo prevalente no sexo feminino e um dos principais contribuintes para redução de mobilidade e perda de independência funcional entre adultos mais velhos. A OA do joelho provoca a destruição da cartilagem articular de forma progressiva, podendo levar à deformidade a região da articulação [Albuquerque et al. 2012, Corti and Rigon 2003].

A etiologia do processo é complexa e inicia-se com o envelhecimento. Porém, alguns fatores durante a vida do paciente podem provocar um início precoce desse processo degenerativo natural, tais como doenças inflamatórias ou infecciosas que destroem a estrutura cartilaginosa, precipitando a osteoartrose [Camanho 2001]. 


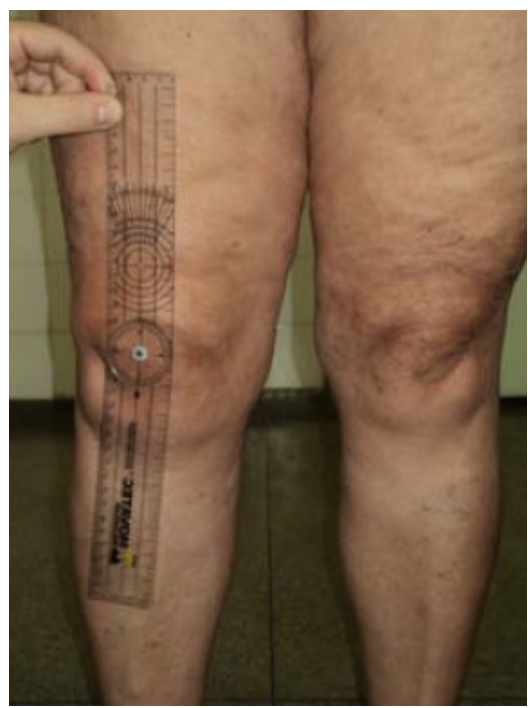

(a) Avaliação clínica.

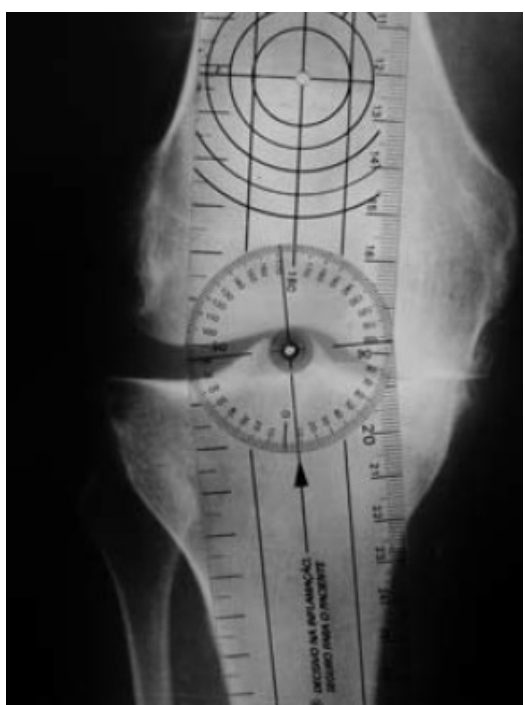

(b) Avaliação radiográfica.

Figura 1. Tipos de mensurações do eixo anatômico do joelho [Albuquerque et al. 2012].

A deformidade articular causada pela OA do joelho é complexa e pode provocar desvios no eixo anatômico. Uma forma clássica de tratamento, para retardar a progressão do processo artrósico e corrigir as deformidades, é a correção cirúrgica do desvio do eixo dos membros inferiores [Camanho 2001].

A radiografia panorâmica dos membros inferiores, que é a incidência radiográfica em todo o membro inferior, incluindo as articulações da bacia, dos joelhos e tornozelos [Santili et al. 1998], em conjunto com apoio mono ou bipodálico, é uma das metodologias mais recomendadas neste tipo de situação de avaliação. Porém, devido a fatores de dificuldades logísticas para efetuação da radiografia panorâmica, um estudo descrito em [Matos et al. 2015] avaliou a concordância entre radiografias panorâmicas e radiografias curtas da região do eixo anatômico do joelho. O estudo conclui que existe concordância fortemente significativa (ICC > 0,96) entre as duas radiografias.

Além da concordância entre a mensuração panorâmica e curta por meio de radiografias, um outro estudo avaliou se há concordância fortemente significativa entre as mensurações clínica (efetuadas diretamente no paciente utilizando um goniômetro) e radiográfica (Figura 1). Os resultados mostram que existe concordância entre os dois métodos de medição, apresentando ICC $=0,974$ [Albuquerque et al. 2012].

A conformidade constatada entre a mensuração clínica e radiográfica abre espaço para estudos que automatizem a medição, tanto de forma clínica, quanto radiográfica, para facilitar o processo de diagnóstico da OA do joelho. Um sistema automático para análise de características do eixo anatômico do joelho por meio de processamento de imagens, descrito em [Oka et al. 2008], o KOACAD (Knee OsteoArthritis Computer-Aided Diagnosis), mede, nas imagens radiográficas, o estreitamento do espaço articular nos lados medial e lateral, a formação de osteófitos e a angulação articular.

Recentes avanços tecnológicos em visão computacional e em outros métodos vêm ganhando espaço na tarefa de auxiliar no processo de visualização e de diagnóstico de 
patologias. Um deles é a Realidade Aumentada (RA), que se caracteriza pela inserção de objetos virtuais no mundo real por meio de um dispositivo tecnológico, aumentando a percepção e interação humana com o mundo real. Ou seja, a utilização de RA possibilita a visualização de informações em objetos que o usuário não consegue ver de forma direta. Sendo assim, é uma tecnologia com grande potencial na área de visualização médica, possibilitando uma interação em tempo real de dados e pacientes reais Azuma 1997, Follmann et al. 2017].

Com o avanço do acesso tecnológico em termos de hardware, recentes lançamentos de dispositivos com tecnologias integradas vêm proporcionando maiores experiências de imersão em realidade aumentada e virtual - como é o caso do Projeto Tango [Google 2017c]. O Projeto Tango é uma plataforma de realidade aumentada e virtual onde sensores de profundidade, reconhecimento de área e posicionamento espacial são integrados a um dispositivo móvel, possibilitando experiências em RA mais precisas, como: navegação interna, mapeamento 3D, mensuração física, entre outras.

A mensuração do eixo anatômico do joelho através de exame radiográfico é ferramenta fundamental no planejamento pré-operatório para realinhamento dos membros inferiores. Contudo, há dificuldades encontradas quanto à logística de aquisição de exame radiográfico de qualidade, aos riscos de exposição do paciente à radiação ionizante e ao custo adicional gerado durante este processo [Matos et al. 2015].

Devido ao cenário exposto, o desenvolvimento e validação de uma alternativa tecnológica que auxilie no processo de mensuração clínica do eixo anatômico do joelho torna-se favorável nos procedimentos de diagnóstico e tratamento da OA do joelho, evitando a exposição dos pacientes ao Raio-X e reforçando o campo de estudo para utilização deste tipo de tecnologia na ortopedia.

O objetivo deste trabalho é descrever o desenvolvimento de uma aplicação clínica de mensuração do eixo anatômico do joelho por meio da utilização de técnicas de RA e análise de imagens de profundidade, para proporcionar ao médico ortopedista o armazenamento da reconstrução 3D da região de interesse para análises posteriores e, por fim, verificar a concordância entre o método desenvolvido e o método radiológico de mensuração.

\section{Metodologia proposta}

Neste trabalho, foi proposto um método para mensuração do eixo anatômico e reconstrução 3D baseados em imagens de profundidade em tempo real. A Figura 2 exibe a sequência de etapas envolvidas nesta tarefa, as quais serão explicadas nas subseções seguintes.

\subsection{Aquisição de imagem}

A captura das imagens RGB e de profudindade dos pacientes em tempo real foi executada utilizando o smartphone Lenovo Phab 2 Pro, dispositivo produzido especialmente para rodar a plataforma Tango [Lenovo 2016]. Possui um processador Qualcomm Snapdragon 652 e uma unidade de processamento gráfico (GPU) para suportar as funcionalidades processadas pelo Tango.

A etapa de aquisição de imagens, na metodologia proposta, dá-se pela utilização da câmera RBG (16 megapixels) e sensor de profundidade do Lenovo Phab 2 Pro. A 


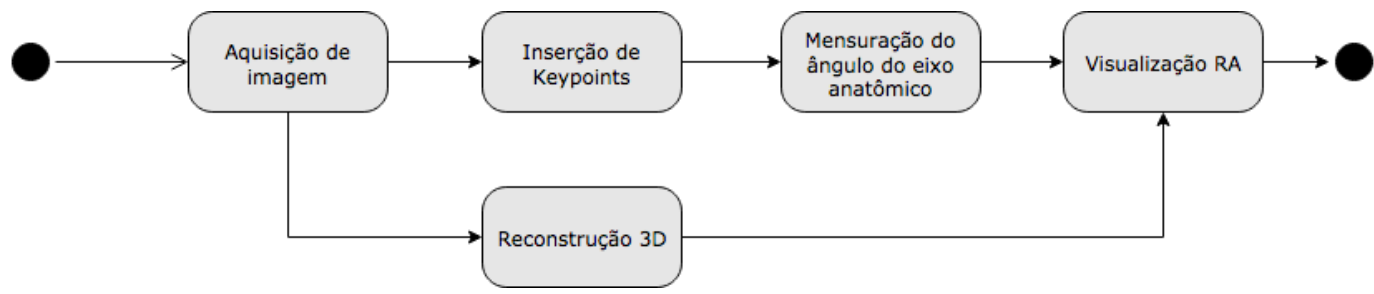

Figura 2. Etapas da abordagem proposta para mensuração do eixo anatômico do joelho com uso de RA.

Figura 3 ilustra as etapas de aquisição de imagem. Primeiramente, tem-se a etapa de captura de vídeo RGB e de profundidade pelo Phab 2, sendo que o paciente em observação encontra-se em pé de frente para o observador com o smartphone. Logo após, os frames são processados pela API do Tango, fornecendo métodos de visualização e manipulação. Por fim, os dados processados são encaminhados para a etapa de processamento, na qual são desenvolvidas as funcionalidades da aplicação.

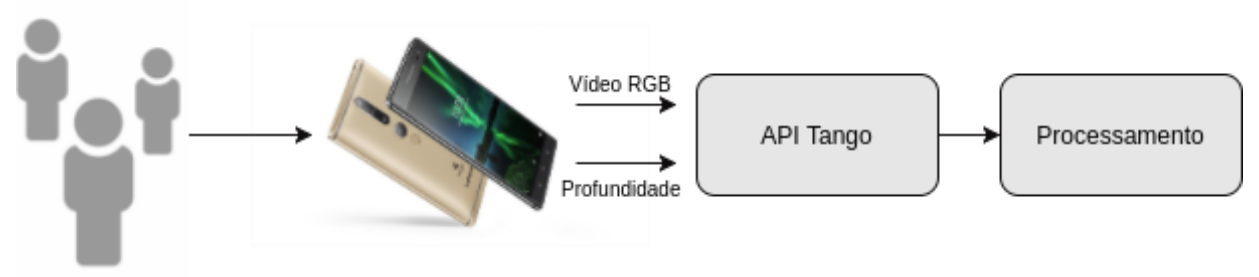

Figura 3. Etapas de aquisição de imagem.

\subsection{Inserção de pontos de interesse}

Para a mensuração do eixo anatômico do joelho, a metodologia proposta necessita de pontos de interesse (POI, do inglês: Point of interest [Sung et al. 2014]) que indiquem o posicionamento do joelho, fêmur e tíbia de cada perna em observação. Sendo assim, no desenvolvimento da aplicação móvel, foi estabelecida uma sequência de inserções de seis POIs que indiquem no paciente a localização dos pontos necessários para mensuração, como mostrado na Figura 4

Cada um destes POIs mapeados pelos keypoints é instanciado interativamente pelo usuário e tridimensionalmente na cena por meio de uma sequência indicada pela aplicação: joelho direito, fêmur direito, tíbia direita, joelho esquerdo, fêmur esquerdo e tíbia esquerda. O sensor de profundidade acoplado ao smartphone em uso permite reconhecer a nuvem de pontos do paciente e sua localização de acordo com um sistema de coordenadas [Google 2017a]. Isto permite que os POIs instanciados sejam independentes do ponto de vista em que a cena está sendo observada.

A Figura 5 ilustra a etapa de inserção virtual dos POIs nas posições correspondentes demonstradas na Figura 4. Na tela da aplicação, um target indica a posição na qual o POI atual a ser mapeado será instanciado tridimensionalmente no mundo.

O funcionalidade de motion tracking que é disponibilizada pela plataforma Tango, faz com que o dispositivo seja capaz de capturar os seus próprios movimentos e orientação em um ambiente 3D [Google 2017b]. Devido a esta característica, o observador tem 


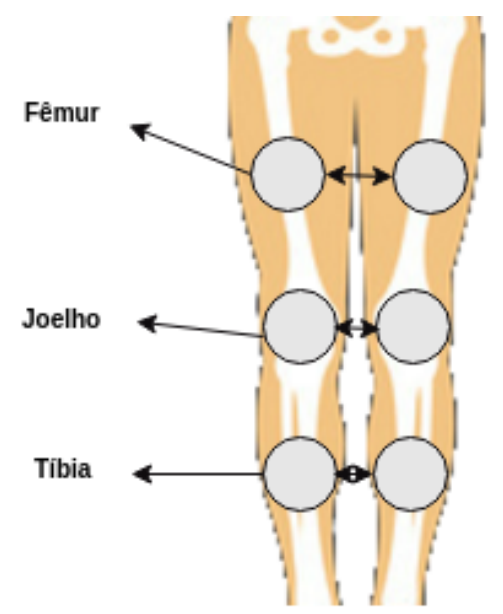

Figura 4. Representação da localização dos pontos necesários para mensuração do ângulo do eixo anatômico do joelho.

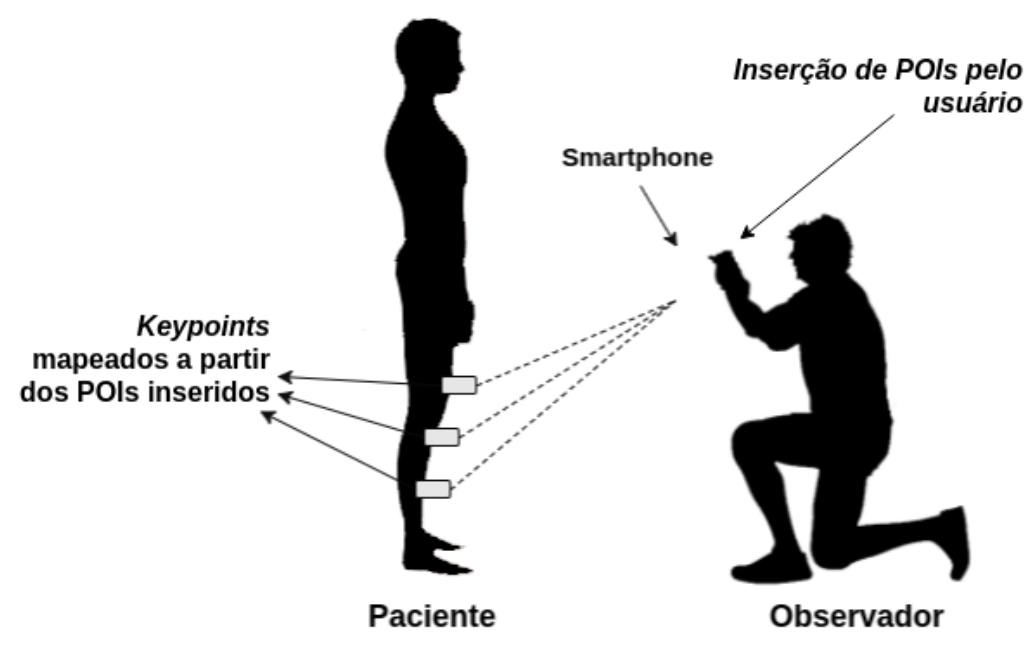

Figura 5. Representação da inserção de keypoints no paciente.

movimentação livre durante o processo de inserção e visualização dos pontos de interesse inseridos. Ou seja, o usuário tem liberdade de se aproximar, se afastar ou se deslocar em relação ao paciente sem que os POIs mapeados anteriormente se desloquem do posicionamento de instância original.

\subsection{Mensuração do ângulo do eixo anatômico}

Os três POIs de cada perna são interpretados como dois vetores, tendo origem no POI do joelho (Figura 6). O ângulo formado entre estes dois vetores $(v 1$ e $v 2)$ é obtido pela Equação 1 .

$$
\Theta=\arccos \left(\frac{\overrightarrow{v 1} \cdot \overrightarrow{v 2}}{\|\vec{v} 1\| \cdot\|\overrightarrow{v 2}\|}\right), 0 \leq \Theta \leq \pi
$$

Por fim, o ângulo obtido é utilizado para orientar o médico o quanto o eixo anatômico deve ser corrigido na cirurgia ortopédica ou indicar o grau de comprometimento em 


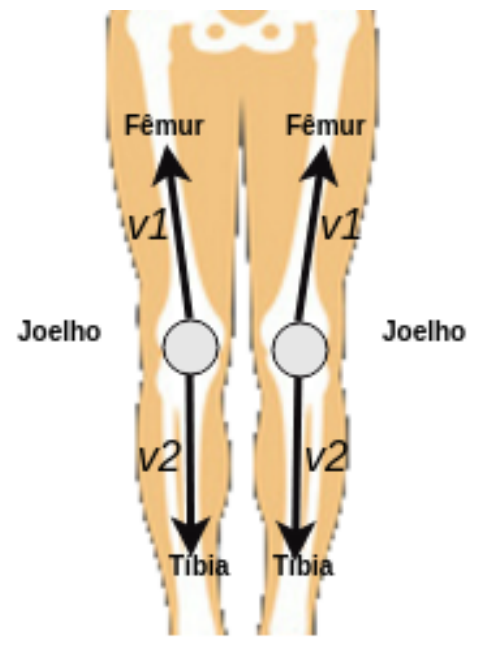

Figura 6. Vetores resultantes dos POIs inseridos.

que se encontra a OA do joelho.

\subsection{Reconstrução 3D}

A API da plataforma Tango oferece diversos métodos para manipulação dos dados obtidos com o sensor de profundidade. Um destes métodos é o de reconstrução 3D baseado na nuvem de pontos gerada pelos raios IR. Esta reconstrução 3D fornecida pela plataforma utiliza as informações de cores capturadas pela câmera RGB e reconstrói todo o mesh em observação.

Na aplicação desenvolvida pela metodologia proposta, o processo de reconstrução 3D acontece desde a inicialização da aplicação. Ou seja, durante o processo de inserção dos POIs e visualização dos resultados, a reconstrução 3D acontece em background. dade de:

O usuário, por meio de botões na interface gráfica da aplicação, tem a possibili-

- visualizar o modelo sendo construído em tempo real, alinhado na posição correspondente ao mundo real;

- pausar/continuar a reconstrução em um determinado momento;

- exportar o modelo obtido no formato OBJ (formato de imagem 3D padronizado).

$\mathrm{Na}$ metodologia proposta, foi adicionado uma filtragem do modelo 3D antes da etapa de exportação ao formato OBJ. Uma vez que o usuário aciona este evento, um bounding box é criado para delimitar quais partes do mesh serão exportados.

Este bouding box, como ilustrado na Figura 7, delimita a região do eixo anatômico do joelho como região válida para exportação, eliminando artefatos indesejados no resultado final (ex: mesas, parede, chão, etc) e incluindo as partes do modelo que se encontra dentro da região delimitada. A região delimitada é calculada a partir do posicionamento dos POIs inseridos pelo usuário, onde a largura $d$ é a distância no eixo $y$ entre o POI do fêmur e o da tíbia. O posicionamento é determinado pelo ponto central no eixo $x$ entre os POIs dos joelhos.

A Figura 8 demonstra o exemplo de uma reconstrução 3D da parte frontal dos eixos anatômicos dos joelhos de um paciente portador de OA. Os modelos são exportados 

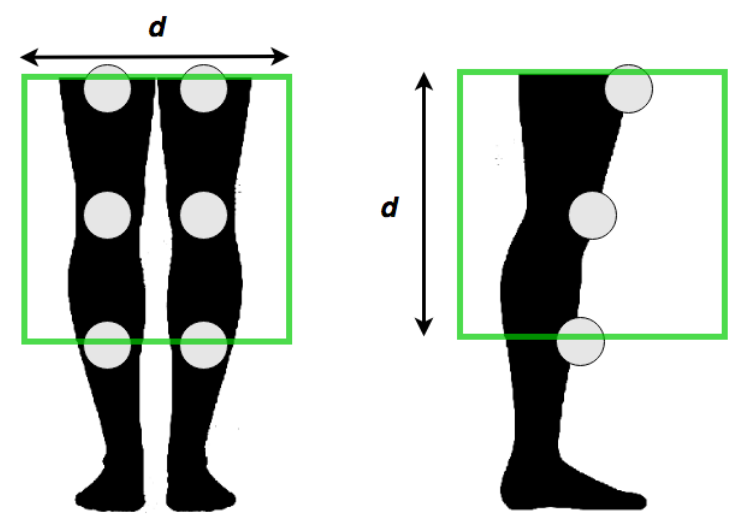

Figura 7. Representação do bounding box que delimita o mesh da região do eixo anatômico do joelho.

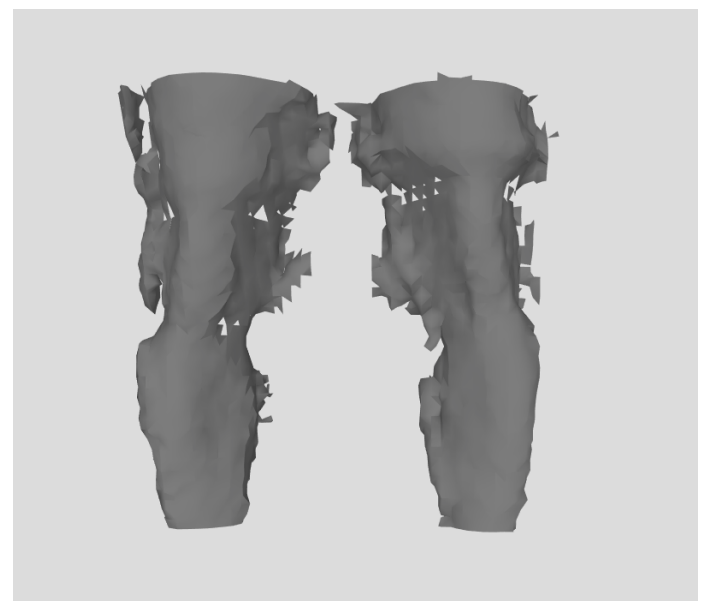

Figura 8. Modelo 3D dos eixos anatômicos dos joelhos de um paciente portador de OA do joelho.

para a memória interna do smartphone e podem ser visualizados por qualquer software de visualização de objetos 3D.

\subsection{Visualização RA}

A visualização final da aplicação desenvolvida insere as informações dos ângulos dos eixos anatômicos no mundo real observado, de forma alinhada e mantendo o motion tracking dos POIs inseridos.

Para cada ângulo obtido, um label contendo o valor do ângulo e o tipo de deformidade é posicionado ao lado do POI referente ao joelho do paciente. O tipo da deformidade é determinado pela direção do ângulo que se forma entre os vetores. Caso o ângulo se apresente na direção externa da perna, classifica-se como joelho valgo, o contrário classifica-se como joelho varo.

A Figura $9 \mathrm{a}$ mostra um screenshot da mensuração dos eixos anatômicos do joelho de um paciente portador de OA. São inseridas linhas entre os pontos referentes a cada 


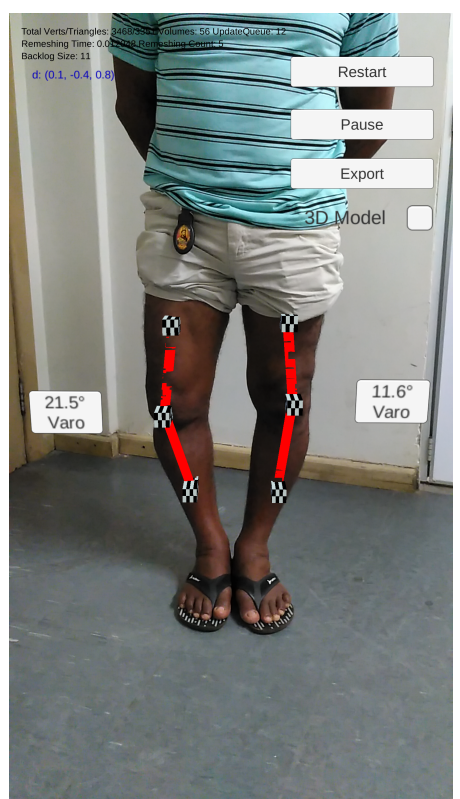

(a) Ângulos obtidos dos eixos anatômicas dos joelhos.

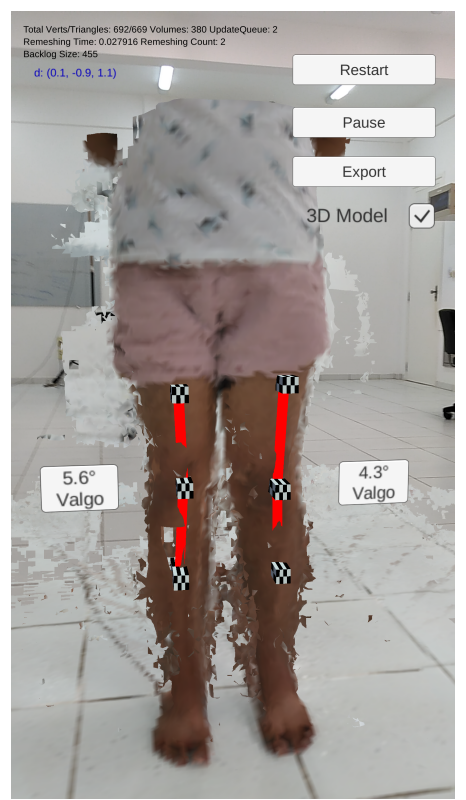

(b) Representação 3D do paciente alinhada ao mundo real.

Figura 9. Visualização das informações de angulos com RA e reconstrução 3D.

perna para facilitar a visualização dos vetores formados.

Por sua vez, a reconstrução 3D possibilita a visualização da representação do paciente mesmo que ele não esteja mais em cena. A ação de pausar a reconstrução faz com que o modelo 3D gerado permaneça inalterável nos frames seguintes, tendo a orientação e posicionamento alinhados ao mundo real pelo motion tracking da plataforma (Figura 9b).

\section{Resultados}

Para obtenção das medidas, o estudo foi realizado no Hospital Universitário da Universidade Federal do Maranhão (HU-UFMA), acompanhado de um médico especialista, membro da Sociedade Brasileira de Cirurgia do Joelho (SBCJ), e residentes do ambulatório de ortopedia. Para fins de avaliação, os resultados são expressos em Coeficiente de Correlação Intraclasse (ICC) entre as medidas, tendo ICC $\geq 0,75$ como valor excelente de reprodutibilidade entre os casos.

Com o propósito de facilitar a comparação entre os métodos, a avaliação baseada em imagens radiográficas será identificada pela sigla MR, enquanto o método proposto neste trabalho será identificado por MRA.

\subsection{Descrição do experimento}

O experimento foi avaliado e aprovado pela Comissão de Ética em Pesquisa (CEP) do HUUFMA, de acordo com a Resolução 466/2012 do Conselho Nacional de Saúde (Diretrizes e Normas Regulamentadoras de Pesquisa Envolvendo Seres Humanos). A aprovação da coleta de dados veio por meio do parecer de número 2.439.948.

Foram analisados 15 pacientes portadores de OA do joelho no período de Dezembro de 2017 a Janeiro de 2018. O total de joelhos mensurados foi 27, pois 3 pacientes não 
possuíam radiografia do joelho esquerdo.

Os pacientes incluídos na pesquisa tinham idade acima dos 18 anos, relataram dores no joelho e ausência de doença reumática nesta articulação, todos atendidos no ambulatório de ortopedia.

A partir das consultas agendadas no ambulatório, os pacientes foram consultados sobre o interesse de participação na pesquisa. O método MR faz parte da rotina ambulatorial para pacientes portadores de OA do joelho. Deste modo, os candidatos que manifestaram interesse em participar do estudo assinaram o Termo de Consentimento Livre e Esclarecido e foram submetidos ao método MRA, proposto neste trabalho.

As imagens radiográficas são de tamanho curto e tiradas com o paciente em posição com carga (paciente em pé, onde o desvio do eixo torna-se mais evidente durante a captura da imagem radiográfica).

Os resultados das mensurações foram então comparados a fim de verificar a concordância entre os métodos em avaliação e determinar a validação do método MRA.

\subsection{Apresentação de resultados e discussão}

Para cada grupo, foram medidos joelhos direito e esquerdo em ambas as técnicas de mensuração. A avaliação através do teste ICC foi feita separadamente para os joelhos direito e esquerdo e uma avaliação de ambos. Para este estudo, o método MR é interpretado como padrão-ouro de mensuração do eixo anatômico.

A Tabela 1 exibe os resultados de ICC para cada grupo de medidas, onde o resultado para o grupo de joelhos direito entre as duas classes apresentou o maior valor, com ICC $=0.98$. Observou-se que existe concordância fortemente significativa entre os métodos MR e MRA em todos os grupos de medida, tendo o valor geral de ICC $=0.972$.

Tabela 1. ICC de cada grupo de medidas

\begin{tabular}{cccc} 
& Joelho Direito & Joelho Esquerdo & Ambos \\
\hline Quantidade & 15 & 12 & 27 \\
ICC & 0.98 & 0.946 & 0.972 \\
\hline
\end{tabular}

Para cada grupo de medidas os valores foram expressados em MSE (do inglês: Mean Squared Error), onde as diferenças de cada grupo (MR - MRA) são elevadas ao quadrado, somadas e divididas pelo total de medições. Enquanto o ICC demonstra o nível de confiabilidade das medições, o MSE mostra mais detalhadamente as diferenças reais entre os métodos em avaliação.

A Tabela 2 traz os valores MSE para os grupos de medição em termos de média, desvio padrão, máximo e mínimo.

Os valores adquiridos com o teste ICC corroboram com os obtidos em [Albuquerque et al. 2012] quanto à reprodutibilidade de mensuração entre as formas radiográfica e clínica. O valor total de ICC $=0.972$ se aproxima de 1 e atesta a homogeneidade dos métodos de medição. Contudo, avaliando os valores ICC de forma separada, as medidas dos joelhos esquerdos adquiridas com o método MRA apresentaram o menor valor $(\mathrm{ICC}=0.946)$. Apesar de ter apresentado o menor MSE $\left(1.76\right.$ graus $\left.^{2}\right)$, teve um des- 
Tabela 2. MSE para cada grupo de medidas

\begin{tabular}{ccccc}
\hline MSE & Média & Desvio Padrão & Máximo & Mínimo \\
\hline Joelho Direito & 1.88 & 1.23 & 4 & 0.09 \\
Joelho Esquerdo & 1.76 & 2.48 & 6.76 & 0.00 \\
Ambos & 1.86 & 1.85 & 6.76 & 0.00 \\
\hline
\end{tabular}

vio padrão maior (2.48 graus $\left.{ }^{2}\right)$ e obteve o maior erro dentre os grupos de medição (6.76 graus $^{2}$ ).

Durante o experimento, foi possível observar que o motion tracking da plataforma Tango, ocasionalmente, desloca os POIs previamente no decorrer da movimentação do smartphone. Este deslocamento acontece para cada POI inserido, o que gera um erro acumulado durante o processo de inserção dos marcadores. Acredita-se que o menor desempenho das medições referentes aos joelhos esquerdos tenha acontecido devido ao fato de que o eixo anatômico esquerdo é o último a ter os POIs inseridos na sequência pré-estabelecida.

Este erro de deslocamento pôde ser bem visualizado durante os testes de alguns candidatos, como foi o caso do paciente da Figura 10 . É possível notar o deslocamento dos POIs após a movimentação do smartphone, dando a impressão de que foram inseridos erradamente. Este deslocamento também acontece durante a sequência de inserção, causando o erro de medição do ângulo devido à diferença de posicionamento inicial definida pelo observador. Apesar disso, os valores estimados pelo método MRA $\left(1.2^{\circ}\right.$ e $\left.13.6^{\circ}\right)$ ainda assim se aproximam dos valores reais obtidos pelo método $\mathrm{MR}\left(0^{\circ}\right.$ e $\left.11^{\circ}\right)$.

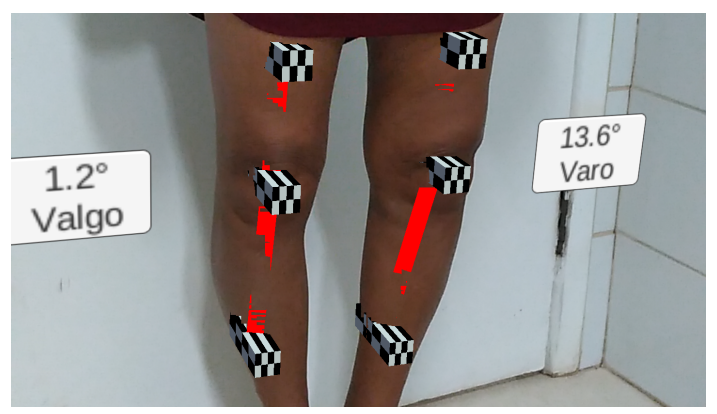

Figura 10. Erro de deslocamento dos POIs (paciente 10).

\subsection{Comparação de resultados}

A última etapa deste trabalho foi comparar os resultados obtidos pelo método proposto com os de alguns trabalhos já mencionados.

A Tabela 3 apresenta os valores de ICC obtidos nos estudos de verificação de correlação entre diferentes métodos de mensuração do eixo anatômico, onde todos os trabalhos apresentam homogeneidade acima de 0.96 .

O estudo desenvolvido em [Matos et al. 2015] verificou concordância fortemente significativa entre a radiografia panorâmica (ideal para este tipo de mensuração) e radiografia curta do eixo anatômico do joelho. Já o estudo proposto em [Albuquerque et al. 2012] verificou a concordância entre a radiografia curta e o método 
clínico. Por sua vez, o trabalho realizado em [Oka et al. 2008] automatiza o processo de mensuração em radiografias curtas, apresentando o maior coeficiente entre os métodos comparados. Finalmente, a metodologia proposta neste trabalho obteve concordância fortemente significativa com mensuração em radiografias curtas e a utilização de RA.

Tabela 3. Comparação de resultados

\begin{tabular}{ccc}
\hline Autores & Classes de mensuração & ICC \\
\hline [Matos et al. 2015] & MR panorâmica e MR curta & 0.96 \\
\hline Albuquerque et al. 2012] & MR curta e medição clínica & 0.974 \\
\hline [Oka et al. 2008] & $\begin{array}{c}\text { MR curta e medição } \\
\text { automática (em radiografia curta) }\end{array}$ & 1 \\
Método proposto & MR curta e medição clínica (MRA) & 0.972 \\
\hline
\end{tabular}

Analisando os coeficientes de correlação entre todas as classes de mensuração apresentadas, é possível afirmar que o método proposto apresenta-se promissor (ICC = 0.972) como alternativa de avaliação dos membros inferiores de pacientes portadores de OA do joelho. Esse método promove redução de custos uma vez que não há necessidade de um exame radiográfico, além da não exposição dos pacientes à radiação ionizante.

\section{Conclusão}

Este trabalho apresentou um método para mensuração do eixo anatômico do joelho de forma clínica com o uso de RA e reconstrução 3D da região de interesse em pacientes portadores de OA. O método proposto utilizou a plataforma Tango, que faz uso de conceitos de RA, visando o desenvolvimento de uma aplicação móvel em que o usuário define a localização de pontos que indiquem os POIs do eixo anatômico a ser analisado, evitando que o paciente seja exposto à radiação ionizante e promovendo redução de custos neste processo.

A fim de validar a utilização do método proposto em situações reais, um grupo de 15 pacientes portadores de OA do joelho foi analisado, sendo que o estudo foi avaliado e aprovado pelo CEP HU-UFMA. Um total de 27 joelhos foram analisados com auxílio da ferramenta desenvolvida e tiveram os resultados comparados (através do teste estatístico ICC) com os números reais obtidos por meio de radiografias curtas com carga. O teste mostrou que os dois métodos tem concordância de $97.2 \%$ nas mensurações obtidas, o que atesta a confiabilidade na utilização do método proposto. Contudo, analisando de forma separada, observou-se que em alguns casos houve uma diferença de até 2.6 graus entre as medições, o que pode limitar a utilização da ferramenta em casos que necessite maior precisão, como no processo pré-operatório.

Dada a concordância averiguada, trabalhos futuros incluem a investigação de uma metodologia de detecção automática dos membros inferiores, eliminando o processo manual de inserção dos POIs. Além disso, pretende-se examinar a diferença de desempenhos entre os joelhos direito e esquerdo e tentar descartar os erros com a detecção automática das articulações. Quanto à reconstrução 3D do eixo anatômico, sugere-se o desenvolvimento de um módulo de visualização na aplicação, na qual o usuário tenha a possibilidade de armazenar um histórico de pacientes e visualize os modelos gerados para cada etapa do processo. 
Espera-se que estas considerações ajudem na elaboração de futuros estudos com a utilização dessa metodologia em pacientes portadores de OA do joelho, e que contribuam para a elaboração de políticas de conscientização e incentivo à prática de utilização da mensuração clínica do eixo anatômico do joelho com o uso de RA.

\section{Referências}

[Albuquerque et al. 2012] Albuquerque, R., Barretto, J., Carvalho, A., Pinheiro, V., Mercante, B., and Assis, D. (2012). Análise comparativa da mensuração do eixo anatómico do joelho. Revista Portuguesa de Ortopedia e Traumatologia, 20(1):81-86.

[Azuma 1997] Azuma, R. T. (1997). A survey of augmented reality. Presence: Teleoperators and Virtual Environments, 6(4):355-385.

[Camanho 2001] Camanho, G. L. (2001). Tratamento da osteoartrose do joelho. Rev Bras Ortop, 36(5):135-40.

[Corti and Rigon 2003] Corti, M. C. and Rigon, C. (2003). Epidemiology of osteoarthritis: Prevalence, risk factors and functional impact. Aging Clinical and Experimental Research, 15(5):359-363.

[Follmann et al. 2017] Follmann, J., Brinhol, G., Tarouco, P., Saraçol, J., and Amaral, É. (2017). Realidade aumentada aplicada ao processo de reabilitação física de membro superior. $17^{\circ}$ Workshop de Informática Médica.

[Google 2017a] Google (2017a). Depth perception I tango I google developers. https: //developers.google.com/tango/overview/depth-perception.

[Google 2017b] Google (2017b). Tango concepts I tango I google developers. https: //developers.google.com/tango/overview/concepts. Acessado em: 10 de janeiro de 2018.

[Google 2017c] Google (2017c). Tango developer overview I tango I google developers.

[Lenovo 2016] Lenovo (2016). Behind our phab 2 pro, the world's 1st tango-enabled smartphone. https://goo.gl/PvH5yP. Acessado em: 10 de janeiro de 2018.

[Matos et al. 2015] Matos, L. F., Giordano, M., Cardoso, G. N., Farias, R. B., Pires, R., et al. (2015). Análise radiográfica comparativa do eixo anatômico na osteoartrite do joelho. avaliação inter e intraobservadores. Revista Brasileira de Ortopedia, 50(3):283-289.

[Oka et al. 2008] Oka, H., Muraki, S., Akune, T., Mabuchi, A., Suzuki, T., Yoshida, H., Yamamoto, S., Nakamura, K., Yoshimura, N., and Kawaguchi, H. (2008). Fully automatic quantification of knee osteoarthritis severity on plain radiographs. Osteoarthritis and Cartilage, 16(11):1300-1306.

[Santili et al. 1998] Santili, C., Waisberg, G., Akkari, M., Fávaros, T., and Prado, J. C. L. (1998). Avaliação das discrepâncias de comprimento dos membros inferiores. Rev Bras Ortop, 33(1):41-4.

[Sung et al. 2014] Sung, M. H., Choi, Y., Ko, H., and Hwang, J. I. (2014). Level-of-detail ar: Managing points of interest for attentive augmented reality. In 2014 IEEE International Conference on Consumer Electronics (ICCE), pages 351-352. 